\title{
ON INTEGRAL ESTIMATES OF NON-NEGATIVE POSITIVE DEFINITE FUNCTIONS
}

\author{
ANDREY EFIMOV, MARCELL GAÁL AND SZILÁRD GY. RÉVÉSZ
}

Abstract. Let $\ell>0$ be arbitrary. We introduce the extremal quantities

$$
G(\ell):=\sup _{f} \frac{\int_{-\ell}^{\ell} f d x}{\int_{-1}^{1} f d x}, \quad C(\ell):=\sup _{f} \sup _{a \in \mathbb{R}} \frac{\int_{a-\ell}^{a+\ell} f d x}{\int_{-1}^{1} f d x},
$$

where the supremum is taken over all not identically zero non-negative positive definite functions. We are interested in the question how large can the above extremal quantities be? This problem was originally posed by Yu. Shteinikov and S. Konyagin for the case $\ell=2$. In this note we obtain exact values for the right limits $\varlimsup_{\varepsilon \rightarrow 0+} G(k+\varepsilon)$ and $\varlimsup_{\varepsilon \rightarrow 0+} C(k+\varepsilon)(k \in \mathbb{N})$, and sufficiently close bounds for other values of $\ell$. We point out that the problem provides an extension of the classical problem of Wiener.

MSC 2000 Subject Classification. Primary 42A82, 42A38, 26 D15.

Keywords and phrases. non-negative positive definite function, Wiener's problem, Schur's theorem, Fourier transform, convolution square

\section{INTRODUCTION}

At first let us fix some notation and basic concepts which will be used throughout the sequel. The symbols $[\cdot]$ and $[\cdot]$ stand for the lower (or in other words, the usual) and the upper integer part, respectively. We recall that a function $f: \mathbb{R} \rightarrow \mathbb{C}$ satisfying

$$
\sum_{i=1}^{n} \sum_{j=1}^{n} c_{i} \overline{c_{j}} f\left(x_{i}-x_{j}\right) \geq 0
$$

for any $n$-tuples $\left(x_{i}\right)_{i=1}^{n} \in \mathbb{R}$ and complex numbers $\left(c_{i}\right)_{i=1}^{n}$ is called positive definite. Positive definiteness of the function $f$ will be denoted by $f \gg 0$. If the function $f$ is positive definite and non-negative in the ordinary sense, then we will say that $f$ is doubly positive which we will also write as $f \ggg 0$. In what follows, the symbol $\star$ stands for the convolution.

The problem, formulated above, was originally posed by S. Konyagin and Yu. Shteinikov, who wanted to use the estimate (in the case $\ell=2$ ) for the paper [13] dealing with number theory. In all our investigations we take the liberty to discuss only the continuous case. We believe that the transfer between the discrete and continuous settings should not cause any difficulty.

This work was supported by the Program for State Support of Leading Scientific Schools of the Russian Federation (project no. NSh-9356.2016.1), by the Competitiveness Enhancement Program of the Ural Federal University (Enactment of the Government of the Russian Federation of March 16, 2013 no. 211, agreement no. 02.A03.21.0006 of August 27, 2013), and by Hungarian Science Foundation Grant \#'s NK-104183, K-109789. The second author was supported by the "Lendület" Program (LP2012-46/2012) of the Hungarian Academy of Sciences and the National Research, Development and Innovation Office - NKFIH Reg. No. K115383. 
We remark that the problem in question is closely related to the celebrated Wiener's problem [12, 16], i.e., the question if in any $L^{p}$ norm and for any fixed $\delta>0$ the ratio

$$
\frac{\int_{-\pi}^{\pi} f^{p}(x) d x}{\int_{-\delta}^{\delta} f^{p}(x) d x}
$$

is bounded for all $2 \pi$ periodical positive definite functions $f$. Clearly, in an appropriate sense this holds for $p=\infty$, as we have $\|f\|_{\infty}=f(0)$ for any positive definite function. Also, it can be proved by means of the Parseval identity that for any given $\delta>0$, we have

$$
\int_{-\pi}^{\pi} f^{2} d x \leq \frac{2 \pi}{\delta} \int_{-\delta}^{\delta} f^{2} d x
$$

whenever $f \in L^{\infty}$, say [12, 16]. This, of course, extends to any even $p=2 m$ powers since if $f$ is positive definite, then so is $f^{m}$. However, it is known that for no other exponents $p \notin 2 \mathbb{N}$ does such a finite bound hold. The first counterexamples were constructed by Wainger [15], and the strongest ones (with arbitrarily large gaps and only idempotent polynomials in place of $f \in L^{p}$ ) can be found in [1].

Furthermore, on the non-compact case of $\mathbb{R}$, any bound between integrals on $[-1,1]$ and $[-k, k]$ must grow to infinity with the length $k$ as $\delta$ is fixed normalized to 1 . This is explained in [8] as "Wiener's property fails with $k \rightarrow \infty$ ". However, the case is similar for $\delta \rightarrow 0$ in the torus $\mathbb{T}:=\mathbb{R} / \mathbb{Z}$ and on the real line $\mathbb{R}$ with $\delta:=1$ and $k \rightarrow \infty$. The ratio in the estimate must depend on the ratio of the corresponding intervals. In this sense, both $\mathbb{T}$ and $\mathbb{R}$ behaves the same: there is a finite upper bound exactly for $p \in 2 \mathbb{N}$ which bound happens to be linear in the ratio of the compared intervals.

At this point let us note that the brave question under study is boldly extending the classical Wiener's problem to the case of $L^{1}$ where it is known to fail in general. The price we pay is that we restrict to doubly positive functions instead of general positive definite functions. However, this is in fact not a restriction but a generalization. Indeed, for any power $p=2 m$, where Wiener's problem has a positive answer, an estimate can be easily deducted from the current setting if we observe the following: for any $f \gg 0$, trivially $f^{2 m} \geq 0$ and also by Schur's theorem $f^{2 m} \gg 0$ whence $f^{2 m} \ggg 0$. Thus the $L^{1}$-problem of Konyagin and Shteinikov can be applied to deduce an answer to Wiener's problem even if there is no Parseval identity at our help in this approach. In other words, the positive answer in the question of Konyagin and Shteinikov sheds light to the fact that somehow the positive cases of Wiener's problem are not so intimately connected to Parseval's formula, while the key now seems to be more of double positivity than any identity.

Konyagin's and Shteinikov's original question was answered positively in [6] where Gorbachev found the following bound. (In fact, this result was originally formulated for the discrete case.)

Theorem 1 (D. V. Gorbachev). For any $f \ggg 0$ and $L>0$ we have

$$
\int_{-2 L}^{2 L} F d x \leq \pi^{2} \int_{-L}^{L} F d x .
$$

In terms of $G(k)$, this result can be reformulated as $G(2) \leq \pi^{2}$. By iterating the above estimate one can obtain some bound for all interval length ratio $\ell$. It happens to be not linear, however, a linear growth might be expected in virtue of the known results in Wiener's problem.

In what follows, we obtain bounds for the whole range of $\ell$ which will be of linear growth. This indeed allows us a direct derivation of the positive answers in Wiener's problem when the 
exponent is $p=2 m$. For the case $k=2$, our upper bound is $G(2) \leq 5$ which is somewhat better than $\pi^{2}$.

\section{The RESUlT}

Let $G(k+0)$ and $C(k+0)$ be the right limits $\overline{\lim }_{\varepsilon \rightarrow 0+} G(k+\varepsilon)$ and $\varlimsup_{\varepsilon \rightarrow 0+} C(k+\varepsilon)$, respectively. We note that both functions $G(\ell)$ and $C(\ell)$ are non-decreasing in $(0, \infty)$, and that $G(\ell) \leq C(\ell)$; also, $C(\ell)=G(\ell)=1$ on $(0,1]$. Our result 11 reads as follows.

Theorem 2. For the extremal constant functions $G(\ell)$ and $C(\ell)$ the following estimates hold.

1. Lower bound. For any $\ell \in \mathbb{R} \backslash \mathbb{N}$, we have $G(\ell), C(\ell) \geq 2[\ell]+1$.

Moreover, for all $k \in \mathbb{N}$, we have $G(k) \geq 2 k-1$ and $C(k) \geq 2 k$.

2. Upper bound. For any $\ell \geq 1$, we have

$$
G(\ell) \leq C(\ell) \leq \frac{1}{2} \frac{([2 \ell]+1)([2 \ell]+2)}{[2 \ell]+1-\ell} \leq\lceil 2 \ell\rceil+1 .
$$

3. Sharpness. As a consequence of the above, our bounds are exact for $G(k+0)$ and $C(k+0)$, i.e., we have $\lim _{\ell \rightarrow k+0} G(\ell)=\lim _{\ell \rightarrow k+0} C(\ell)=2 k+1$ for all $k \in \mathbb{N}$.

We remark that instead of the space of doubly positive functions, we could consider the space of smooth doubly positive functions or only measurable doubly positive functions. However, the constants would not differ essentially.

The proof of Theorem 2 is composed of two lemmas. Before presenting them, let us explain the idea implemented in Lemma 1 since the actual formulas may hide it a little.

Our strategy is the following. We consider the so-called periodically extended Dirac delta, that is, $\Phi:=\sum_{k=-\infty}^{\infty} \delta_{k p}$. This "function" is obviously non-negative and positive definite since it can be regarded as the characteristic function of a group, namely, the discrete group $p \mathbb{Z}$. Here the period $p$ is chosen to be $1+\varepsilon$ in order to minimize the presence of values $p z(z \in \mathbb{Z})$ in the segment $[-1,1]$, but at the same time make these values as densely occurring in other intervals as possible. It is easy to show that $\int_{-1}^{1} \Phi d x=1$ and, for any given length $k p<\ell<(k+1) p$, we will have $\int_{-\ell}^{\ell} \Phi d x=2 k+1$ and $\int_{-0}^{\ell-0} \Phi d x=k+1$. The only technical matter is to make this construction fitting into the class of doubly positive functions. We will do this below.

Lemma 1. For all $k \in \mathbb{N}$ we have $G(k+0) \geq 2 k+1$. Moreover, $C(k) \geq 2 k$.

Proof. Let us fix $k \in \mathbb{N}$ and $\varepsilon>0$. We are to estimate $C(k)$ and $G(k+\varepsilon)$ from below.

Let $f_{n}(x):=\cos ^{2 n}\left(\frac{\pi}{p} x\right)$ where $1 \leq p<1.1$ and $n$ is assumed to be large enough. Clearly, for all values of the parameters $n$ and $p$ the function $f_{n}$ is doubly positive and $p$-periodic. It is easy to see that with any given fixed value of $\delta \in(0,0.1)$,

$$
\frac{\int_{-\delta}^{\delta} f_{n}(x) d x}{\int_{-p / 2}^{p / 2} f_{n}(x) d x} \rightarrow 1 \quad(n \rightarrow+\infty)
$$

\footnotetext{
${ }^{1}$ We are indebted to Prof. V. Bogachev, who nicely disproved our initial and naive guess that perhaps even $C(1)=1$, i.e. $\int_{a-1}^{a+1} f \leq \int_{-1}^{1} f(f \gg 0)$ could hold.

Actually, he took $f$ to be the probability density function arising from of a convolution square of some symmetric probability distribution. Then $f$ will be symmetric, non-negative and positive definite. It is clearly possible that at some point $L>0 f$ has $f^{\prime}(L)>0$, whence by symmetry also $f(-L)=f(L)$ and $f^{\prime}(-L)=-f^{\prime}(L)$. Now clearly if $\Phi(a):=\int_{a}^{a+2 L} f$, then $\Phi^{\prime}(a)=f(a+2 L)-f(a)$ and $\Phi "(a)=f^{\prime}(a+2 L)-f^{\prime}(a)$, whence for a small change $\delta>0$ we must have $\Phi(-L+\delta)-\Phi(-L) \approx \Phi^{\prime}(-L) \delta+\Phi^{\prime \prime}(-L) \delta^{2} / 2=f^{\prime}(L) \delta^{2}>0$, and so with $a=-L+\delta$ the proposed inequality fails.
} 
i.e., the function $f_{n}$ is concentrated in the segment $(-\delta, \delta)$ with respect to the period (in the limit, when $n \rightarrow \infty)$. Thus, we see that $f_{n}$ is concentrated in $\cup_{m \in \mathbb{N}} \Omega_{m}$ where $\Omega_{m}:=(-\delta+m p, \delta+m p)$.

To make estimates for $\int_{-k-\varepsilon}^{k+\varepsilon} f_{n}(x) d x$ for some $0<\varepsilon<1$, we have to find how many segments $\Omega_{m}$ are contained in the intervals $[-k-\varepsilon, k+\varepsilon]$. If we chose $\delta<p-1$, then the interval $[-1,1]$ contains only one of $\Omega_{m}$, namely $\Omega_{0}$, and is disjoint from the rest.

Now if we take $\delta$ small enough, and $p$ sufficiently close to 1 (more exactly, if $k(p-1)+\delta<\varepsilon$ ), then the interval $[-k-\varepsilon, k+\varepsilon]$ already contains all $\Omega_{m}$ with $-k \leq m \leq k$. Thus $G(k+\varepsilon) \geq$ $(2 k+1)$, whence also $G(k+0) \geq 2 k+1$.

Furthermore, the inequality $C(k) \geq 2 k$ can be easily seen from considering e.g. the interval $[1,2 k+1]$ which contains $\Omega_{m}$ for all $m=1, \ldots, 2 k$ whenever $2 k(p-1)+\delta<1$.

As the functions $G(\ell)$ and $C(\ell)$ are both non-decreasing, and $C(\ell) \geq G(\ell)$, we obtain the first lower bound of Theorem 2. As for $G(k)$, clearly we have $G(1)=1$, while for $k>1$ we can easily use monotonicity of $G$ to derive $G(k) \geq G(k-1+0) \geq 2 k-1$. The other estimate $C(k) \geq 2 k$ is contained in the above Lemma, whence Part 1 of Theorem 2 is proved.

Lemma 2. We have for any $\ell>1$ the inequality

$$
C(\ell) \leq \frac{1}{2} \frac{([2 \ell]+1)([2 \ell]+2)}{[2 \ell]+1-\ell} .
$$

Proof. Fix the interval $I:=[-1 / 2,1 / 2]$. For temporary use let us denote by $\chi:=\chi_{I}$ the characteristic function of $I$, and we denote by $\chi_{a}(x):=\chi(x-a)$ for indices $a \in \mathbb{R}$. We shall also use the triangle function $T:=\chi \star \chi=(1-|x|)_{+}\left(\right.$where $\left.\xi_{+}:=\max (\xi, 0)\right)$ which is an important example of a non-negative positive definite function.

Consider the functions $g_{a}:=\chi-\chi_{a}$ and $h_{a}:=g_{a} \star \widetilde{g_{a}}$, where $\widetilde{g_{a}}(x):=\overline{g_{a}(-x)}$. So, in view of $g_{a}$ being a real-valued function, then $\widetilde{g_{a}}(x)=g_{a}(-x)=\chi(x)-\chi_{-a}(x)$. Then obviously $h_{a}(x)=T(x)-T(x+a)-T(x-a)+T(x)=2 T(x)-(T(x+a)+T(x-a))$. Because $h$ is defined as a convolution square, it is obvious that $h_{a} \gg 0$ for any $a \in \mathbb{R}$.

Let us involve here an additional parameter $p$ with $0<p \leq 1$. Take now the sum $H_{a, k, p}:=$ $\sum_{j=0}^{k} h_{a+j(2-p)}$ with some $k \in \mathbb{N}$. Then we can estimate $H_{a, k, p}$ the following way:

$$
\begin{aligned}
H_{a, k, p}(x) & :=\sum_{j=0}^{k} h_{a+j(2-p)}(x) \\
& =2(k+1) T(x)-\left(\sum_{j=0}^{k} T(x+a+j(2-p))+\sum_{j=0}^{k} T(x-a-j(2-p))\right) \\
& \leq 2(k+1) \chi_{[-1,1]}(x)-p\left(\chi_{[a-1+p, a+k(2-p)+1-p]}(x)+\chi_{[-a-k(2-p)-1+p, 1-a-p]}(x)\right) .
\end{aligned}
$$

Note that $H \gg 0$ together with its summands $h_{a+j(2-p)}$. Multiplying by any (say, continuous) doubly positive function $f$ we get by an application of Schur's theorem $H f \gg 0$, whence

$$
0 \leq \widehat{H f}(0)=\int_{-\infty}^{\infty} H f \leq 2(k+1) \int_{-1}^{1} f(x) d x-2 p \int_{a+p-1}^{a+k(2-p)+1-p} f(x) d x
$$

using also that $f$, as a positive definite real-valued function, is necessarily even.

Let now $b:=a+p-1$. It follows that

$$
p \quad \int_{b}^{b+k(2-p)+2-2 p} f(x) d x \leq(k+1) \int_{-1}^{1} f(x) d x .
$$


That is, in terms of $C(\ell)$,

$$
C\left(\frac{(k+1)(2-p)-p}{2}\right) \leq \frac{k+1}{p}
$$

So let us take an arbitrary $\ell>1$, and write it in the form $\ell=\frac{(k+1)(2-p)-p}{2}$ with suitable values of $k$ and $p$. This is equivalent to $p=\frac{2(k+1)-2 \ell}{k+2}$. Further, the double inequality $0<p \leq 1$ is equivalent to $\ell-1<k \leq 2 \ell$, whence for $C(\ell)$ we obtain that the estimate

$$
C(\ell) \leq \frac{1}{2} \frac{(k+1)(k+2)}{k+1-\ell}
$$

holds true for any integer $k$ between $\ell$ and $2 \ell$ (and with the respective choice of parameter $p$ ). So, it remains to minimize estimate (3) in the range $k \in(\ell-1,2 \ell]$ for any fixed $\ell>1$.

In order to do so, consider the auxiliary functions

$$
\varphi:(\ell-1,2 \ell] \rightarrow \mathbb{R}, \quad \varphi(x):=\frac{(x+1)(x+2)}{x+1-\ell}=x+\ell+2+\frac{\ell(\ell+1)}{x+1-\ell}
$$

and

$$
\psi:(\ell, 2 \ell] \rightarrow \mathbb{R}, \quad \psi(x):=\varphi(x)-\varphi(x-1) .
$$

Straightforward computation gives us that $\psi(x)=0$ is equivalent to

$$
\frac{(x+1)(x+2)}{x+1-\ell}=\frac{x(x+1)}{(x-\ell)}
$$

the unique solution of which on the interval $(\ell, 2 \ell]$ being $x=2 \ell$. Since $\psi$ is continuous we deduce that the sign of the function $\psi$ does not vary on the interior of its domain. Further, as $\ell>1$, we find

$$
\psi(\ell+1)=\varphi(\ell+1)-\varphi(\ell)=1-\frac{\ell(\ell+1)}{2}<0 .
$$

This yields that the function $\psi$ is negative in $(\ell, 2 \ell)$, whence $\varphi$ is non-increasing on the set of integers in $(\ell-1,2 \ell]$. Therefore, the minimum of $\varphi$ is certainly achieved at the unique integer in $(2 \ell-1,2 \ell]$, in other words at $[2 \ell]$. S, we substitute $k=[2 \ell]$ in (3) which indeed yields the desired inequality (21).

The last inequality in (1) can be obtained easily considering separately the cases where $2 \ell=$ $m \in \mathbb{N}$ (providing equality), and where $2 \ell \notin \mathbb{N}$ (leading to strict inequality).

As in the above argument concerning the lower bound, the proof of the upper estimate will be completed adding that $G(\ell) \leq C(\ell)$, always.

Finally, we are in a position to prove Part 3, that is, the sharpness statement.

The inequality $G(k+0) \geq 2 k+1$ is clear, because the lower estimate in Part 1 provides $G(k+\varepsilon) \geq[2(k+\varepsilon)]+1=2 k+1$ for arbitrary $\varepsilon>0$. Moreover, from Lemma 2 we also get

$$
\begin{gathered}
C(k+0)=\lim _{\varepsilon \rightarrow+0} C(k+\varepsilon) \leq \frac{1}{2} \frac{([2 k+2 \varepsilon]+1)([2 k+2 \varepsilon]+2)}{[2 k+2 \varepsilon]+1-(k+\varepsilon)}= \\
\lim _{\varepsilon \rightarrow+0} \frac{1}{2} \frac{(2 k+1)(2 k+2)}{2 k+1-(k+\varepsilon)} \leq 2 k+1 .
\end{gathered}
$$

Altogether, we have $2 k+1 \leq G(k+0) \leq C(k+0) \leq 2 k+1$ and equality holds everywhere, as needed.

Therefore, the proof of Theorem 2 is complete. 


\section{Concluding Remarks}

Let us see what general framework for the construction of the above estimates and proofs can be set up. Basically, what we do is to look for an auxiliary function $H$, positive definite itself, and satisfying

$$
H \leq A \chi_{[-1,1]}-B \chi_{[a, a+\ell]}-B \chi_{[-a-\ell,-a]}
$$

or

$$
H \leq A \chi_{[-1,1]}-B \chi_{[-\ell, \ell]}
$$

By Schur's theorem we also have that $f H \gg 0$ for any $f \gg 0$, whence

$$
0 \leq \widehat{f H}(0)=\int_{-\infty}^{\infty} f H \leq A \int_{-1}^{1} f-2 B \int_{a}^{a+\ell} f
$$

and $C(\ell / 2) \leq A / 2 B$, or

$$
0 \leq \widehat{f H}(0)=\int_{-\infty}^{\infty} f H \leq A \int_{-1}^{1} f-B \int_{-\ell}^{\ell} f
$$

and $G(\ell) \leq A / B$.

Let $\ell>0$ be arbitrary. Let us now consider the extremal quantities

$$
\sigma(a, \ell):=\inf \left\{\frac{A}{2 B}: \exists H \gg 0, H \leq A \chi_{[-1,1]}-B \chi_{[a, a+\ell]}-B \chi_{[-a-\ell,-a]}\right\}, \sigma(\ell):=\sup _{a \in \mathbb{R}} \sigma(a, \ell),
$$

and

$$
\gamma(\ell):=2 \sigma(0, \ell)=\inf \left\{\frac{A}{B}: \exists H \gg 0, H \leq A \chi_{[-1,1]}-B \chi_{[-\ell, \ell]}\right\} .
$$

Clearly, from the above it follows that we have $C(\ell / 2) \leq \sigma(\ell)$ and $G(\ell) \leq \gamma(\ell)$, always. Let us make a few additional remarks here. First, the setting here is quite general, but at least in $\mathbb{R}$ any "reasonable" positive definite function $H$ can be represented as a "convolution-square": $H=$ $G \star \widetilde{G}$, of say some $G \in L^{2}$ function, see e.g. [5]. The construction of $H:=H_{a, k, p}$ worked somehow along different lines, for we instead represented $H$ as the sum of other convolution squares with well-controlled supports: but in principle the direct convolution square representation is also possible.

The above defined extremal problems $\sigma$ and $\gamma$ are very much like the so-called Turán or Delsarte extremal problems. The main difference is that here we want to compare integrals over given intervals to integrals over given central pieces, while in the Turán and Delsarte problems we normalize with respect to $f(0)$ and compare to this normalization either the full integral, or (in case of the so-called "pointwise Turán problem") a particular one-point value. In the recent work [8], more concrete application of the Turán and Delsarte problems are worked out for the case of Wiener's problem in several dimensions. This type of approach seems to be reasonable here, too.

Since for $H \gg 0$ we necessarily have $0<\widehat{H}(0)=\int H$, it follows immediately that $A \geq B \ell$, whence $\gamma(\ell) \geq \ell$ and $\sigma(\ell) \geq \ell / 2$. But in the virtue of our lower estimations of $C(\ell)$ and $G(\ell)$, it is apparent that these are far from being sharp. From the other side, it could be well that we would have $\sigma(\ell)=C(\ell / 2)$ and $\gamma(\ell)=G(\ell)$. The essential part of the above constructions (i.e. the ones for the upper estimation) targeted the computation (or estimation) of $\gamma(\ell)$ and $\sigma(\ell)$. We conjecture that in principle this approach is best possible.

The interested reader can consult for further details about the Turán and Delsarte problems and their applications in e.g. packing problems in [2], [3], [4], [7], [9], [10], [11] and [14]. 


\section{REFERENCES}

[1] A. Bonami, Sz. Gy. RÉvÉsz, Failure of Wiener's property for positive definite periodic functions. $C$. $R$. Math. Acad. Sci. Paris, 346 (2008), no. 1-2, 39-44.

[2] H. Cohn, New upper bounds on sphere packings. II, Geom. Topol. 6 (2002), 329-353.

[3] H. Cohn, N. Elkies, New upper bounds on sphere packings. I, Ann. of Math. (2) 157 (2003), no. 2, 689-714.

[4] H. Cohn, A. Kumar, S. Miller, D. Radchenko, M. Viazov, The sphere packing problem in dimension 24. Preprint, arXiv:1603.06518.

[5] W. Ehm, T. Gneiting, D. Richards, Convolution roots of radial positive definite functions with compact support. Trans. Amer. Math. Soc. 356 (2004) no. 11, 4655-4685.

[6] D. V. Gorbachev, Certain inequalities for discrete, nonnengative, positive definite functions (in Russian) Izvestiya Tulskogo Universiteta Estestvennie Nauki 2015 no. 2, 1-8.

[7] D. V. Gorbachev, Extremal problem for entire functions of exponential spherical type, connected with the Levenshtein bound on the sphere packing density in $\mathbb{R}^{n}$ (in Russian), Izvestiya of the Tula State University Ser. Mathematics Mechanics Informatics 6 (2000) 71-78.

[8] D. V. Gorbachev, S. Yu. Tikhonov, Wiener's problem for positive definite functions. Perprint, arXiv:1604.01302,

[9] Ivanov, V. I., On the Turán and Delsarte problems for periodic positive-definite functions. (Russian) Mat. Zametki 80 (2006), no. 6, 934-939; translation in Math. Notes 80 (2006), no. 5-6, 875-880.

[10] Sz. Gy. RÉvÉsz, Extremal problems for positive definite functions and polynomials. Thesis for the degree Doctor of the Academy, pp. 164 (2009), Budapest.

[11] Sz. Gy. RÉvÉsz, Turán's extremal problem on locally compact abelian groups, Anal. Math. 37 (2011) no. $1,15-50$.

[12] Shapiro, H. S., Majorant problems for Fourier coefficients, Quart. J. Math. Oxford Ser. (2) 26 (1975), 9-18.

[13] Yu. N. Shteinikov, On the Set of Joint Representatives of Two Congruence Classes Trudy Matematicheskogo Instituta imeni V.A. Steklova (in Russian) 290 (2015), p. 202-210. English version: Proceedings of the Steklov Institute of Mathematics, Vol. 290, (2015), pp. 189-196.

[14] M. Viazovska, The sphere packing problem in dimension 8. Preprint, arXiv:1603.04246.

[15] Wainger, S, A problem of Wiener and the failure of a principle for Fourier series with positive coefficients, Proc. Amer. Math. Soc. 20(1969) 16-18.

[16] N. Wiener, A class of gap theorems, Ann. Scuola Norm. Sup. Pisa (2) 3 (1934), 367-372.

ANDREY EFIMOV

URAl Federal University

620000 Ekaterinburg, PR. Lenina 51., RUSSiA

E-mail address: anothar@ya.ru

Marcell GaÁl

Bolyai Institute, University of Szeged

6720 Szeged, Aradi vérTanúk tere 1., HUNGARY

AND

MTA-DE "Lendület" Functional Analysis Research Group

Institute of Mathematics, University of Debrecen

4010 Debrecen, PO. Box 12, HUNGARY

E-mail address: marcell.gaal.91@gmail.com

SzILÁRD Gy. RÉvÉSZ

Institute of Mathematics, Faculty of Sciences

BudAPEST University of TECHNOLOGY AND ECONOMICS

1111 Budapest, MứEgYetem RKP. 3-9., HUNGARY

AND

A. RÉnyi Institute of Mathematics

Hungarian ACADEMy of Sciences,

1053 Budapest, ReÁltanoda UtCa 13-15., HUNGARY

E-mail address: revesz.szilard@renyi.mta.hu 